Draft version March 12, 2018

Preprint typeset using $\mathrm{LAT}_{\mathrm{E}} \mathrm{X}$ style emulateapj v. 5/2/11

\title{
PROBING THE STRUCTURE OF THE OUTFLOW IN THE TIDAL DISRUPTION FLARE SW J1644+57 WITH LONG-TERM RADIO EMISSION
}

\author{
Di $\mathrm{CAO}^{1,2}$, XIANG-YU WANG ${ }^{1,2}$ \\ Draft version March 12, 2018
}

\begin{abstract}
The recently discovered high-energy transient $\mathrm{Sw} \mathrm{J} 1644+57$ is thought to arise from the tidal disruption of a passing star by a dormant massive black hole. The long-term, bright radio emission of $\mathrm{Sw} \mathrm{J} 1644+57$ is believed to result from the synchrotron emission of the blast wave produced by an outflow expanding into the surrounding medium. Using the detailed multi-epoch radio spectral data, we are able to determine the total number of radiating electrons in the outflow at different times, and further the evolution of the cross section of the outflow with time. We find that the outflow gradually transits from a conical jet to a cylindrical one at later times. The transition may be due to collimation of the outflow by the pressure of the shocked jet cocoon that forms while the outflow is propagating in the ambient medium. Since cylindrical jets usually exist in AGNs and extragalactic jets, this may provide independent evidence that Sw J1644+57 signals the onset of an AGN.
\end{abstract}

Subject headings: galaxies: nuclei- gamma-rays: bursts

\section{INTRODUCTION}

The high-energy transient Swift J164449.3+573451 (hereafter Sw J1644+57) was detected by Swift Burst Alert Telescope (BAT) on March 28, 2011 (Burrows et al. 2011). It was considered as a gamma-ray burst (GRB) in the beginning. However, Sw J1644+57 has later shown many different characters from GRBs and from active galactic nuclei (AGNs) as well. It has a lifetime much longer than GRBs and is much more luminous than AGNs. Further observations show that the position of this event is coincident with the nucleus of a galaxy at redshift $z \simeq 0.3534$ (Levan et al. 2011; Bloom et al. 2011). Its long-term X-ray luminosity varies as $L_{X} \propto t^{-5 / 3}$, consistent with the expected fallback rate of the tidally disrupted material (Rees 1998). Thus this event was later considered as a star being tidally disrupted by a $\sim 10^{6} M_{\odot}$ supermassive black hole $(\mathrm{SMBH})$ in the center of the galaxy. Since the $\gamma$-ray and X-ray luminosity of this event is 2-3 orders of magnitude larger than the Eddington luminosity of a $\sim 10^{6} M_{\odot} \mathrm{SMBH}$, the emission is likely to be produced by a collimated outflow. It is worth noting that Sw J1644+57 has a bright long term radio synchrotron emission (Zauderer et al. 2011), which provide a unique chance for us to study how the physical parameters of the outflow evolve as it interacts with the ambient medium.

Metzger et al. (2012) modeled the radio-microwave emission during 5-23 days after the trigger with the blast wave emission, in analogy to GRB afterglows (see e.g. Giannios \& Metzger 2011). They find that a narrow jet of an opening angle of $\theta_{j} \lesssim 1 / \gamma_{j}$ expanding into a medium with a density profile $n \propto R^{-2}$ could fit the radio data. Berger et al. (2012) reconsidered this model based on much longer radio data during 5-216 days. They noticed that the energy injection model used in Metzger

\footnotetext{
${ }^{1}$ Department of Astronomy, Nanjing University, Nanjing, 210093, China

${ }^{2}$ Key laboratory of Modern Astronomy and Astrophysics (Nanjing University), Ministry of Education, Nanjing 210093, China
}

et al.(2011) is no longer suitable after 23 days. Instead they made an assumption that the energy distribution in the ejecta is described by $E(>\Gamma) \propto \Gamma^{-2.5}$ (where $\Gamma$ is the bulk Lorentz factor of the ejecta), so that substantial energy is added into the blast wave at later times. The radial density profile after 23 days is inferred to be $n \propto R^{-1.5}$ with a plateau at $r \approx 0.4-0.6 \mathrm{pc}$. Both works made a pre-assumption that the jet has a conical structure with a constant opening angle $\theta_{j} \lesssim 1 / \gamma_{j}$. However the jet structure of this transient has not been resolved yet. The cross sections of extragalactic AGN radio jets do not increase at large radii and cylindrical jet structure (or blob) has been usually assumed (Bridle \& Perley 1984). Since Sw J1644+57 involves a jet from a $\mathrm{SMBH}$ similar to an AGN, it is necessary to consider the possibility of a cylindrical jet3. In this paper we first present an approach to calculate the parameters of the jet blast wave at any instantaneous time directly by using the radiation spectrum at that time (§2). This approach applies to both conical and cylindrical jets. Then, using these parameters, we calculate the jet cross section of $\mathrm{Sw}$ $\mathrm{J} 1644+57$ at different times and study how it evolves as the jet propagates outward $(\S 3)$. We find that the outflow gradually transits from a conical jet to a cylindrical one at later times. The possible mechanism for the collimation is further discussed (§3.2). Finally, we give our conclusions $(\S 4)$.

\section{MODELING THE RADIO EMISSION}

In our model, the radio afterglow of $\mathrm{Sw} \mathrm{J} 1644+57$ is produced by electrons accelerated by the forward shock that forms as the jet interacts with the ambient medium. The radiation can be simply described by a cloud of relativistic electrons radiating synchrotron emission in the magnetic fields, so it is completely determined by the following parameters: the total number of radiating electrons $N_{e}$, the bulk motion Lorentz factor $\Gamma$, the magnetic field $B$, the thermal Lorentz factor of electrons $\gamma_{e}$ and

3 The possibility of cylindrical jets has also been discussed for GRBs (Cheng et al. 2001; Wang et al. 2005). 
power-law distribution index $p$. We made the same assumption that $\theta_{j} \lesssim 1 / \gamma_{j}$ in the conical jet case, so the parameter $\theta_{j}$ is not involved. The bulk Lorentz factor relates with the jet energy $E_{j}$ through

$$
\Gamma=\left(\frac{E_{j}}{m_{p} N_{e} c^{2}}\right)^{\frac{1}{2}} .
$$

Assuming that the magnetic field energy density is a factor $\varepsilon_{B}$ of the shock internal energy, the magnetic field related with the number density of the ambient medium $n$ through

$$
B=\Gamma c\left(32 \pi n m_{p} \varepsilon_{B}\right)^{\frac{1}{2}} .
$$

The minimum energy of the accelerated power-law electrons is given by

$$
\gamma_{m}=\varepsilon_{e} \frac{m_{p}}{m_{e}} \frac{(p-2)}{(p-1)} \Gamma,
$$

where $\varepsilon_{e}$ is the equipartition factors of electron energy density.

The radio synchrotron emission produced by the power-law electrons can be described by three characteristic quantities, i.e. the typical frequency $\nu_{m}$, the selfabsorption frequency $\nu_{a}$ and the peak flux $F_{\nu_{\mathrm{m}}}$ (Sari et al. 1998). The two break frequencies are, respectively, given by

$$
\nu_{m}=\frac{\Gamma \gamma_{m}^{2} e B}{2 \pi m_{e} c}
$$

and

$$
\nu_{a}=\nu_{m}\left(C_{0}(p-1) \frac{e n R}{B \gamma_{m}^{5}}\right)^{\frac{3}{5}}
$$

where $C_{0} \approx 10.4 \frac{p+2}{p+2 / 3}$ (Wu et al. 2005). The emission radius $R$ is given by

$$
R=\frac{2 \Gamma^{2} c t}{1+z}
$$

where $t$ is the observer time.

In a relativistic cylindrical jet, the radiation emitted by electrons is distributed over a solid angle of $2 \pi / \Gamma^{2}$. The peak flux can be obtained by calculating the total emission of radiating electrons $N_{e} P_{\nu, \max }$, where $P_{\nu, \max }$ is the peak spectral power (Sari et al. 1998), so the observed peak flux density is

$$
F_{\nu_{\mathrm{m}}}=\frac{N_{e} P_{\nu, \max }(1+z)}{2 \pi D_{L}^{2} / \Gamma^{2}} .
$$

On the other hand, for a conical jet with $\theta_{j} \lesssim 1 / \Gamma$, the observed peak flux density can be written as:

$$
F_{\nu_{\mathrm{m}}}=\frac{N_{e, i s o} P_{\nu, \max }(1+z)}{4 \pi D_{L}^{2}} \frac{\Gamma^{2} \theta_{j}^{2}}{2},
$$

where $N_{e, i s o}$ is the equivalent isotropic electron number, which relates with the real number of electrons $N_{e}$ by

$$
N_{e} \simeq N_{e, i s o} \frac{\theta_{j}^{2}}{4}
$$

Substituting $N_{e, \text { iso }}$ in equation (9) into equation (8), we find that the expressions for the peak flux are the same for both cylindrical and conical jet models. Thus equations (4)-(7) apply to both cylindrical and conical jets.

Equations (4), (5) and (7) can be rewritten as explicit expressions of five independent parameters $\varepsilon_{B}, \varepsilon_{e}, N_{e}$, $n$, and $E_{j}$, i.e.

$$
\begin{gathered}
F_{\nu_{\mathrm{m}}}=C_{F} \varepsilon_{B}^{\frac{1}{2}} n^{\frac{1}{2}} E_{j}^{2} N_{e}^{-1}, \\
\nu_{a}=C_{a} \varepsilon_{e}^{-1} \varepsilon_{B}^{\frac{1}{5}} n^{\frac{4}{5}} E_{j}^{\frac{4}{5}} N_{e}^{-\frac{4}{5}}, \\
\nu_{m}=C_{m} \varepsilon_{e}^{2} \varepsilon_{B}^{\frac{1}{2}} n^{\frac{1}{2}} E_{j}^{2} N_{e}^{-2},
\end{gathered}
$$

where $C_{F}, C_{a}, C_{m}$ are three constant coefficients. After setting typical values for $\varepsilon_{B}$ and $\varepsilon_{e}$ (for instance, $\varepsilon_{B}=$ 0.1 and $\varepsilon_{e}=0.1$; Panaitescu \& Kumar 2000), we can solve the other three quantities $N_{e}, n, E_{j}$, using the radio spectral data at different times.

Once we know the evolution of $N_{e}, n, E_{j}$ with the observer time $t$, we can obtain $\Gamma(t), R(t)$, and $n(R)$ with the assistance of equations (1) and (6).

\section{PARAMETER EVOLUTION OF SW J1644+57}

Now we confront the above model with the observational data of Sw J1644+57. We adopt the observational radio data of $\mathrm{Sw} \mathrm{J} 1644+57$ from Berger et al. (2012). Fig. 1 shows our fit of the radio spectral data with the synchrotron broken power-law. We find that the favorable value for the power-law index is $p \simeq 2.4$. The self-absorption frequency $\nu_{a}$ is always below the typical frequency $\nu_{m}$ during the observation period. Table 1 summarizes the characteristic quantities of the broken power-law spectra at different times for Sw J1644+57. With these data, we can get the characteristic parameters, such as $\Gamma, n$ and $R$, at different times without assuming the blast wave dynamics beforehand, i.e. we do not need to assume the ambient density profile or energy injection mode.

Because the spectral data during 36-68 days are incomplete, we could only get the spectral quantities $\nu_{a}$ and $F_{\nu_{\mathrm{a}}}$. With these quantities the jet energy $E_{j}$ can be obtained precisely, while we can only get upper limits for the ambient density $n$ and the total number of radiating electrons $N_{e}$. However, as will be mentioned below, the Lorentz factor of the ejecta may be constant during 23216 days. If we take the assumption that the Lorentz factor during 36-68 days is a constant, e.g. $\Gamma \simeq 3$, the values of $n$ and $N_{e}$ can be obtained. We show the evolution of the parameter $E_{j}(t), n(R), R(t)$ and $\Gamma(t)$ in figure 2 .

\subsection{Evolution of the jet energy and the density profile of the ambient medium}

The jet energy of Sw J1644+57 evolves as $E_{j} \propto t^{0.6}$ during 5-23 days (Fig. 2(a)), $E_{j} \propto t^{1}$ at later times, and after 126 days $E_{j} \propto t^{0.6}$ again. This indicates that Sw J1644+57 has a continuous energy injection lasting much longer than previous thought. The Swift XRT data of Sw J1644+57 obey the power-law decay $L_{x} \sim t^{-5 / 3}$. Such a slow energy injection is insufficient to account for the required energy increase, so an extra or new energy supply mechanism should be needed. In figure 2(b) we can see that the ambient density profile varies as $n \propto$ $R^{-(1.3-1.5)}$, which is consistent with the prediction by 
the Bondi accretion as suggested by Berger et al. (2012). The radius of the jet displayed in figure 2(c) evolves as $R \propto t^{1.1}$ during 23-216 days and $R \propto t^{0.7}$ during 5-23 days. Since $R \propto \Gamma^{2} t$, this relation indicates that the Lorentz factor of the ejecta varies as $\Gamma \propto t^{-0.15}$ during 5-23 days and remains almost constant $\left(\Gamma \propto t^{0.05}\right)$ during 23-216 days. This behavior is clearly seen in figure 2(d). This is the reason why we assume $\Gamma \simeq 3$ during 36-68 days in the calculation.

\subsection{Jet cross section evolution}

The above equations apply to both conical and cylindrical jets. However, the structure could be known if we know how the cross section of the jet evolves as the radius $R$ increases. The size of the cross section can be derived from the evolution of $N_{e}, n$ and $R$. Since the total number of swept-up electrons by the jet relates with the cross section radius through

$$
N_{e}(R)=\int \pi r_{j}^{2}(R) n(R) d R
$$

we can derive the cross section radius by

$$
r_{j}(R)=\sqrt{\frac{1}{\pi n(R)} \frac{d N_{e}}{d R}} .
$$

To obtain $\frac{d N_{e}}{d R}$, we first find an empirical function to fit the relation between $N_{e}$ and $R$, and then calculate the differential coefficient. The result is shown in figure 3 . We find that when $R$ is smaller than a few $10^{18} \mathrm{~cm}$, the jet cross section evolved as a conical jet with $r_{j} \propto R$, which is consistent with the result obtained by Metzger et al. (2012). However at larger radii $\left(R \gtrsim 4 \times 10^{18} \mathrm{~cm}\right)$, $r_{j}$ increase slowly and it tends to become a constant (i.e. $r_{j} \sim$ constant), which indicates that the jet may transit to a cylindrical structure.

The transition may be due to the collimation of the jet by the surrounding cocoon which forms while the jet is propagating in the ambient medium (Bromberg et al. 2011). Such a mechanism has been discussed in longlived AGN jets (Begelman \& Cioffi 1989), as well as in microquasar jets and GRB jets (Bromberg et al. 2011). In this mechanism, matter in the jet head is heated and flows sideways due to its higher pressure than the ambient medium, which leads to the formation of a pressured cocoon around the jet. If the cocoon pressure is sufficiently high, it collimates the jet and reduces its opening angle. Thus a jet can be conical initially and transit to a cylindrical jet at later times. Bromberg et al. (2011) find the condition under which the jet will be collimated:

$$
\widetilde{L} \simeq \frac{L_{j}}{\Sigma_{j} \rho_{\alpha} c^{3}} \lesssim \theta_{0}^{-4 / 3},
$$

where $\widetilde{L}$ is a dimensionless parameter that defines the ratio between the energy density of the jet and the restmass energy density of the surrounding medium at the location of the head, $L_{j}$ is the jet luminosity, $\Sigma_{j}$ is the jet cross section, $\rho_{\alpha}$ is the ambient medium density, and $\theta_{0}$ is the initial opening angle of jet. This condition gives a critic transition radius at

$$
R_{c} \gtrsim \sqrt{\frac{L_{j}}{\pi_{j} \theta_{0}^{2 / 3}{ }^{3}}},
$$

above which the jet becomesacollinated and transits to a cylindrical jet.

For the case of $\mathrm{Sw} \mathrm{J} 1644+57$, we can obtain the critic transition radius by taking $L_{j}=d E_{j} / d t, \rho_{\alpha}=n m_{p}$ and assuming a typical initial opening angle of $\theta_{0}=10^{\circ}$. Figure 4 shows the critic radius $R_{c}$ as a function of time (the dotted line). It clearly shows the critic radius approaches the jet radius at late times, which means that jet tends to be collimated at later times. The transition radius is $\sim 5-8 \times 10^{18} \mathrm{~cm}$, which roughly (within a factor of a few) agrees with the above result obtained by modeling of the radio spectral data (Fig. 3). The fact that the jet in a tidal disruption event transits from conical to cylindrical structure in a way similar to long-lived AGN jets provides independent support that Sw J1644+57 may result from the onset of an AGN.

\section{CONCLUSIONS}

The structure of the jets in tidal disrupted flares such as $\mathrm{Sw} \mathrm{J} 1644+57$, whether it is conical as usually assumed in GRB jets or cylindrical as seen in some extragalactic radio jets, is largely unknown. We propose that the longterm radio data can be used to probe the jet structure. By fitting the observed radio data of Sw J1644+57, we find that the jet structure of $\mathrm{Sw} \mathrm{J} 1644+57$ undergos a transition from a conical jet to a cylindrical one at tens of days after the initial flare. It is natural to expect that the jets are initially conical and later become cylindrical due to collimation by the surrounding cocoon. Observations of extragalactic radio AGN jets show that the jets are indeed cylindrical on large scales. The similar processes occurring in AGNs and Sw J1644+57 may possibly indicate that $\mathrm{Sw} \mathrm{J} 1644+57$ also arise from accretion process in a supermassive black hole, likely feeded by the tidally captured stellar material.

We thank Rongfeng Shen for useful discussions and the referee for the constructive report. This work is supported by the 973 program under grant 2009CB824800, the NSFC under grants 10973008, 11273016 and 11033002, the Excellent Youth Foundation of Jiangsu Province and the Fok Ying Tung Education Foundation.

\section{REFERENCES}

Berger, E. et al. 2012, ApJ, 748, 36

Beskin, V. S. 1997, Phys.-Uspekhi, 40 (7), 659

Bloom, J. S. et al. 2011, Science, 333, 203

Bridle, A. H., \& Perley, R. A. 1984, ARA\&A, 22, 319

Bromberg, O. et al. 2011, Apj, 740, 100

Burrows, D. N. et al. 2011, Nature, 476, 421

Cheng, K. S., Huang, Y. F. \& Lu, T. 2001, MNRAS, 325, 599
Giannios, D. \& Metzger, B. D. 2011, MNRAS, 416, 2102

Levan, A. J. et al. 2011, Science, 333, 199

Metzger, B. D. et al. 2012, MNRAS, 420, 3528

Panaitescu, A. \& Kumar, P. 2000, ApJ, 543, 66

Rees, Martin J. 1988, Nature, 333, 523

Sari, R., Piran, T. \& Narayan, R. 1998, ApJ, 497L, 17

Wang, X. Y., Cheng K. S. \& Tam P. H. 2005, ApJ, 621, 894 
TABLE 1

The Radio Spectrum fit of Sw J1644+57. Data during 5-23 Days ARe taken from Metzger et Al.(2012). The Rest are obtained FROM the DATA of BERGER ET AL. (2012) BY USING THE BROKEN POWER-LAW SPECTRAL FIT.

\begin{tabular}{lllll}
\hline \hline$t($ day $)$ & $\nu_{a}(\mathrm{GHz})$ & $\nu_{m}(\mathrm{GHz})$ & $F_{\nu_{\mathrm{m}}}(\mathrm{mJy})$ & $F_{\nu_{\mathrm{a}}}(\mathrm{mJy})$ \\
\hline 5 & 40 & 386 & 28.6 & - \\
23 & 11.6 & 50.9 & 15.3 & - \\
36 & 8.12 & - & - & 10.56 \\
51 & 9.34 & - & - & 12.93 \\
68 & 8.87 & - & - & 17.08 \\
95 & 8.15 & 23.8 & 31.3 & - \\
126.5 & 7.42 & 23.6 & 30.3 & - \\
161 & 6.04 & 18.2 & 28.2 & - \\
197 & 5.38 & 15.3 & 26.5 & - \\
216 & 5.89 & 17.9 & 26.2 & - \\
\hline
\end{tabular}
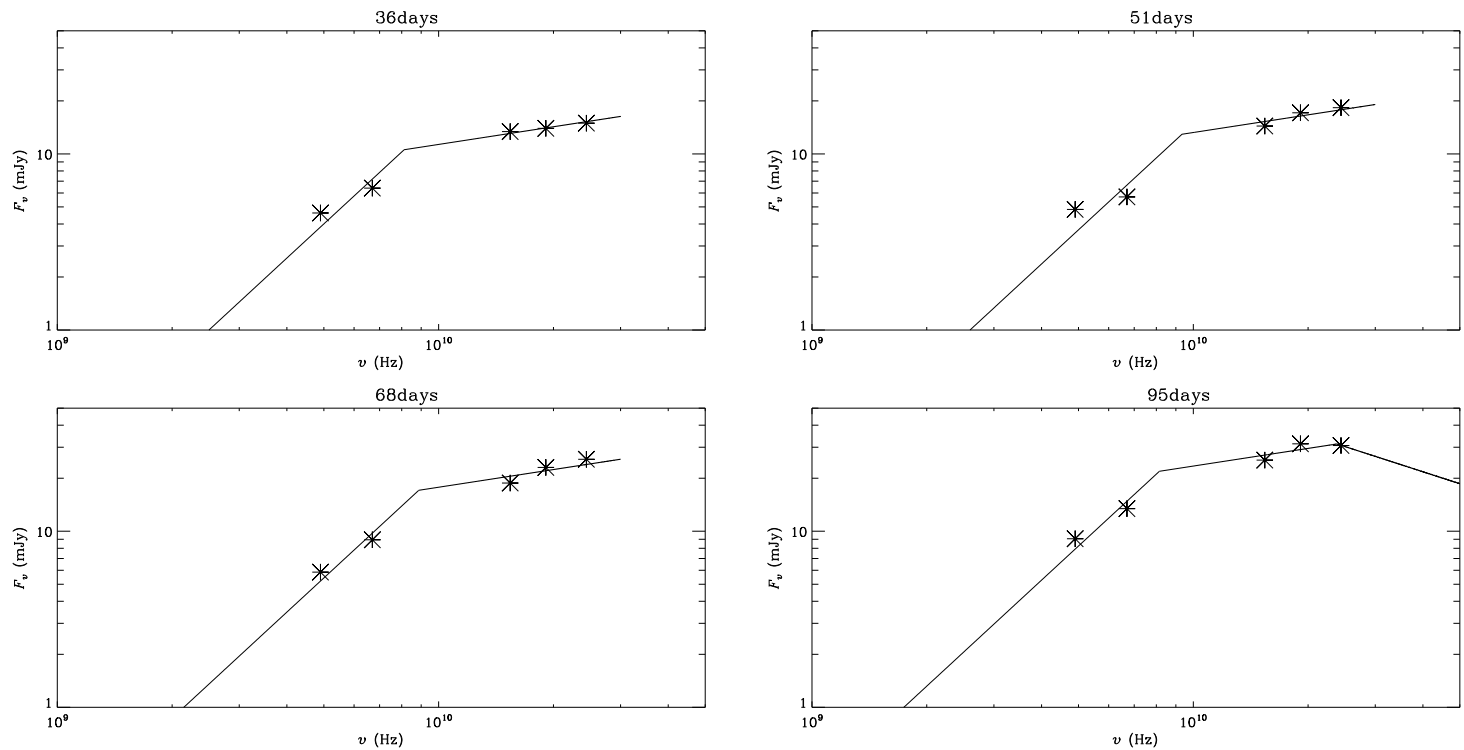

127 days
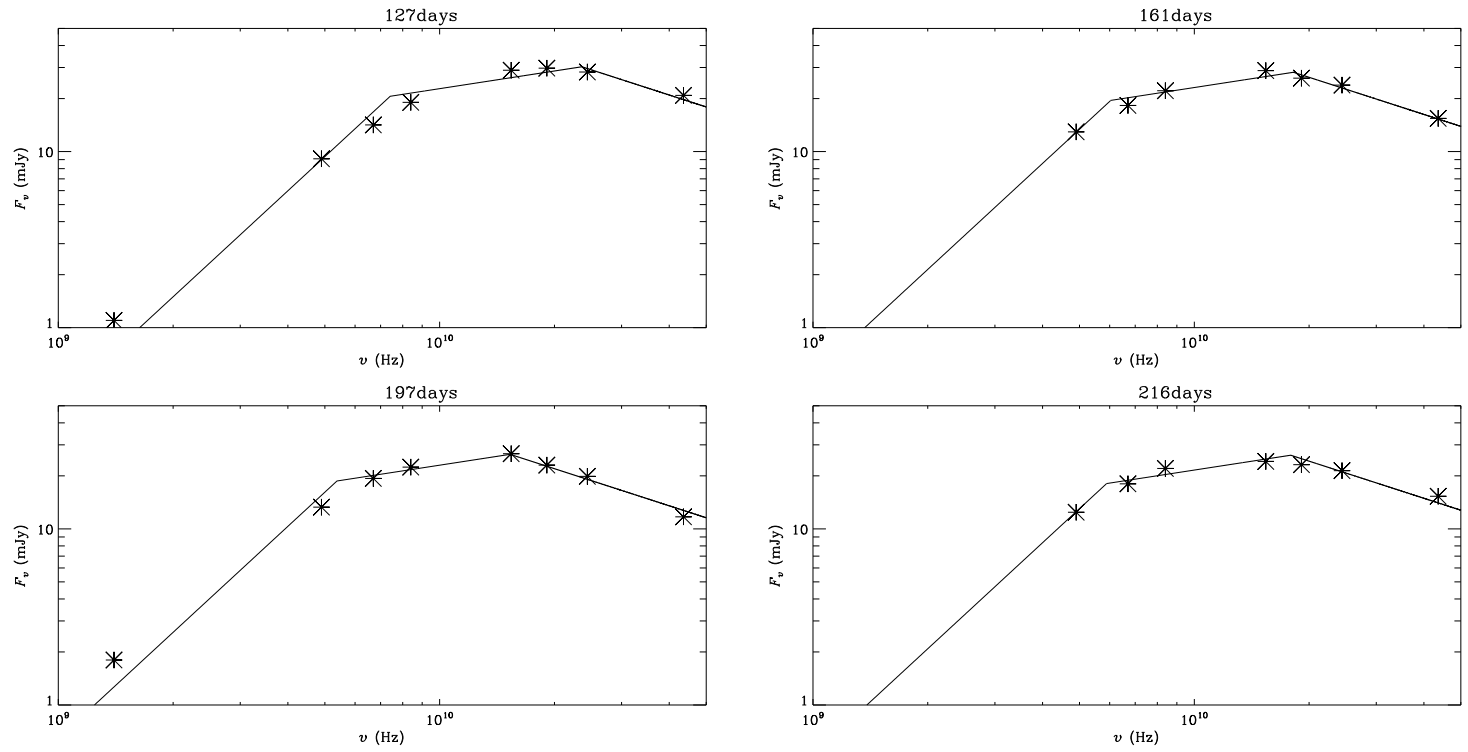

FIG. 1. - The best fit of the radio data of Sw J1644+57 using the synchrotron broken power-law spectra. Observation data are taken from Berger et al. (2012)

$\mathrm{Wu}, \mathrm{X} . \mathrm{F}$. et al. 2005, ApJ, 619, 968

Zauderer, B. A. et al. 2011, Nature, 476, 425 
(a)

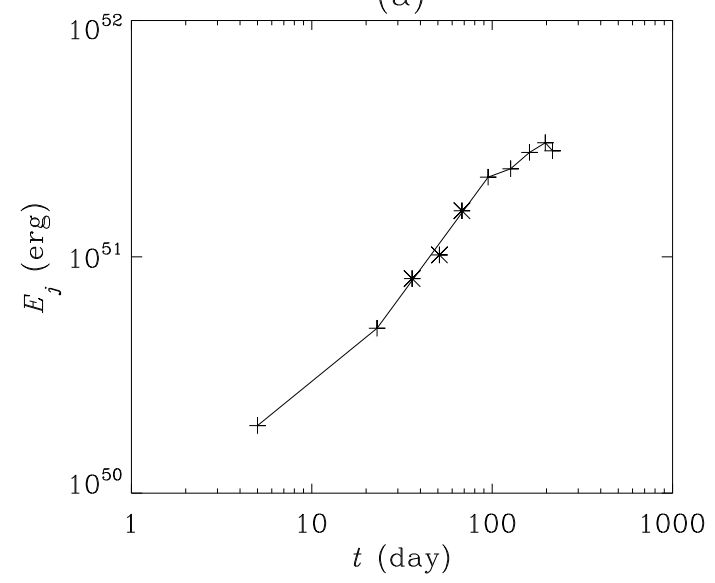

(c)

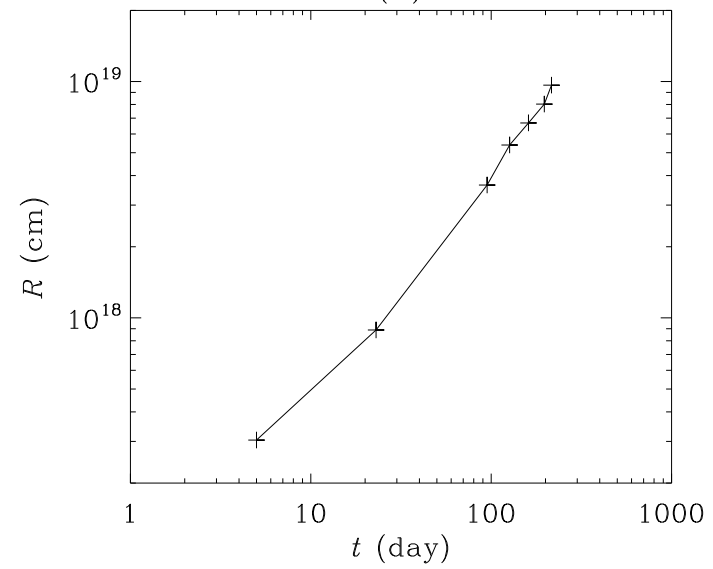

(b)

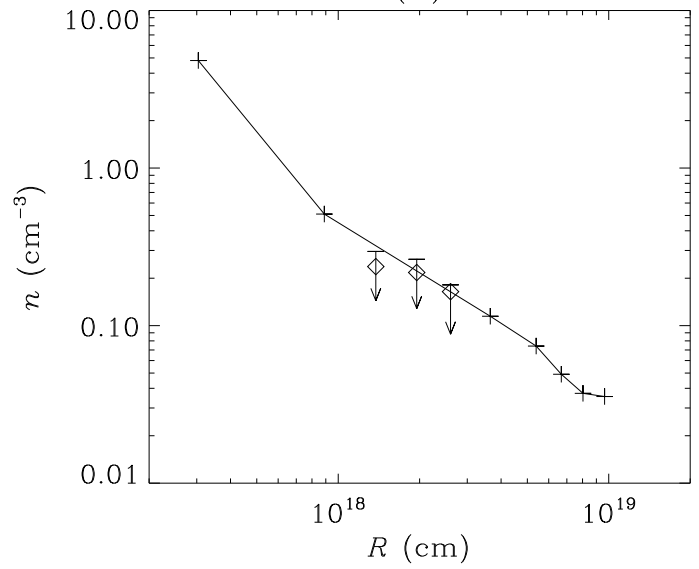

(d)

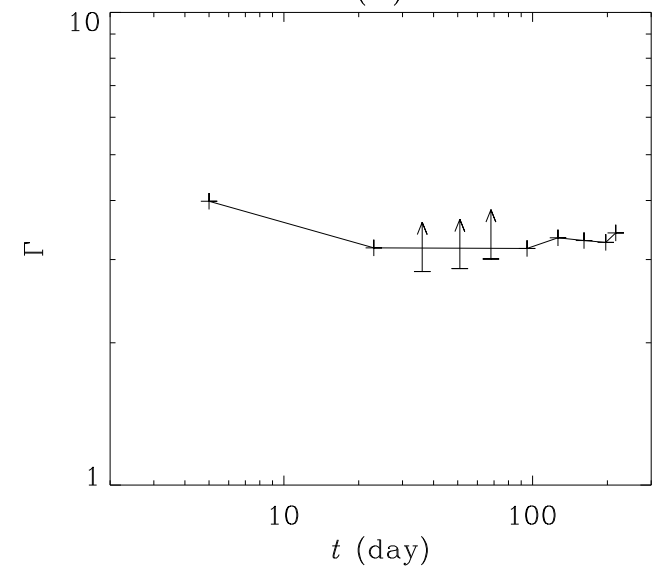

FIG. 2.- Time-evolution of the parameters $E_{j}, n, R$ and $\Gamma$, corresponding to panels (a)-(d). The equipartition factors of electron energy density and magnetic density are $\varepsilon_{e}=0.1$ and $\varepsilon_{B}=0.1$ respectively. The power-law index is $p=2.4$. Due to the incompleteness of radio data during 36-68 days, we plot the parameters obtained during this period with different symbols. The star symbol represents data calculated by using $\nu_{a}$ and $F_{\nu_{\mathrm{a}}}$ only. The diamond symbol represents the values of the number density $n$ derived by using the assumption $\Gamma=3.16$. 


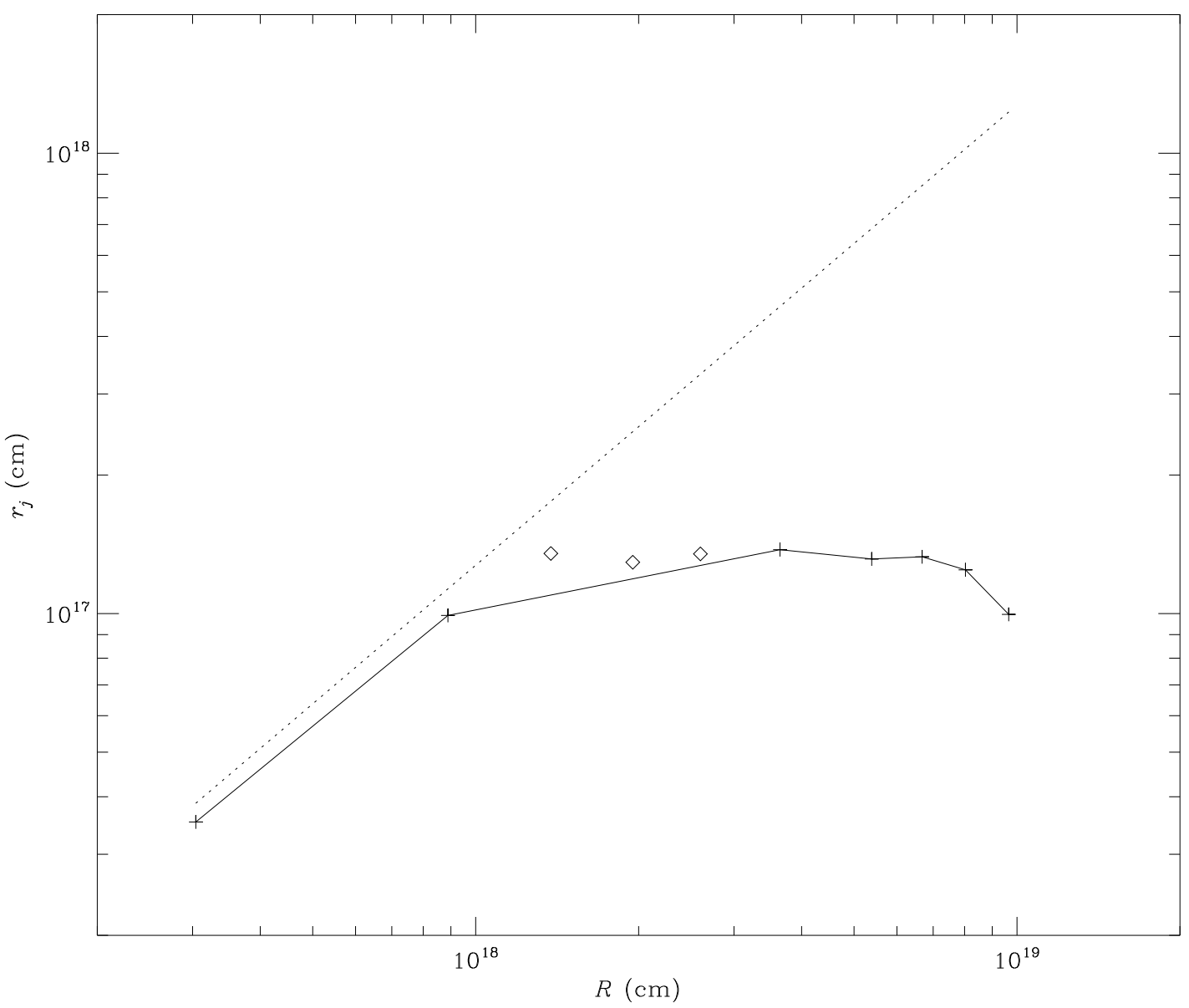

Fig. 3.- Evolution of jet cross section radius $r_{j}$. The dotted line exhibits the expect evolution of $r_{j}$ for the conical jet model. The solid line shows the evolution of $r_{j}$ derived from the radio data. 


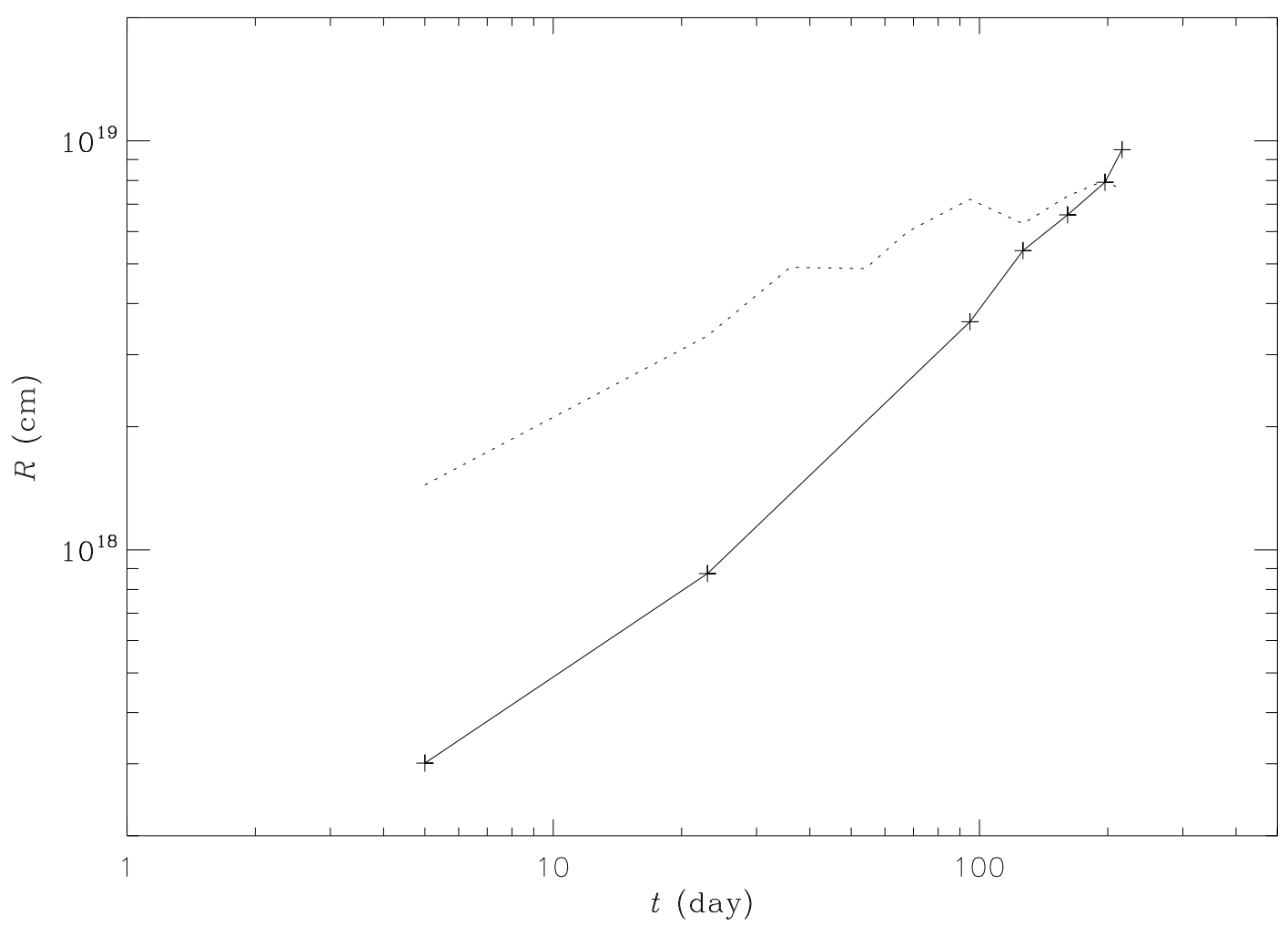

FIG. 4.- The dotted line denotes the critic radius of jet collimation for the parameters of Sw J1644+57, obtained with the condition found by Bromberg et al (2011). The solid line shows the evolution of the jet radius $R$ inferred from the observed radio data. 